\title{
Nonlinear Material Based All-Optical Parallel Subtraction Scheme: an Implementation
}

\author{
Samir Sahu ${ }^{1, *}$, Radha Raman Pal ${ }^{1}$, Shantanu Dhar ${ }^{2}$ \\ ${ }^{1}$ Department of Physics and Technophysics, Vidyasagar University, Midnapore, 721102, India \\ ${ }^{2}$ Department of Physics, Jhargram Raj College, Jhargram, 721507, India
}

\begin{abstract}
Non-linear material based all-optical switching mechanism is utilized here to implement the all-optical parallel subtraction scheme. Optical tree architectures here convert analog optical signal to the corresponding digital one. A two bit all-optical binary parallel subtractor has been proposed and which may be elevated to a higher bit parallel subtractor in course using all-optical half-subtractor and a full-subtractor. These are constructed by a composite slab of linear medium (LM) and non-linear medium (NLM) and it is the building block of our proposed subtructor circuit. These circuits are all-optical and fully parallel in nature. It can also gear up to the highest ability of optical performance in high-speed all-optical computing system.
\end{abstract}

Keywords Nonlinear Material, All-Optical Switch, Optical Tree Architecture, All-Optical Logic Gate, All-Optical Parallel Subtractor

\section{Introduction}

As our modern life is becoming faster day by day, in the current decade, scientific and engineering problems are specially confined in a particular area to attain the reliable, faithful and high speed performances in computation[1-3,5] and communication[4,6]. Unfortunately, the VLSI technology, so far is being used, cannot beat this challenge. Electrons have a limited speed. So, this technology is already in a saturated state from both the aspects - size and speed. Moreover, our venture for the advancement of this technology creates new problems like hot carriers, electromagnetic interference, short channel effects etc. These factors endanger the device reliability.

Presently, in fewer fields, electronic devices are replaced by electro-optical (OE) devices[6,11]. This technology temporarily overcomes some physical problems. But the previous limitations still exist as electronic parts are still there in those hybrid components.

In the above circumstances, there is a way-out to replace the traditional circuits with all-optical ones[2-3,7,15-17]. Photonics is the future of technology. In the last few years, light, as signal carrier, has already established its validity in various all-optical operations[1-3,5-7,9,11,15-23]. Among many other techniques, non linear material (NLM) has been established its validity as optical switching devices[1-3,5,9].

* Corresponding author:

tosamirsahu@gmail.com (Samir Sahu)

Published online at http://journal.sapub.org/ijoe

Copyright (C) 2011 Scientific \& Academic Publishing. All Rights Reserved
All-optical signal processing technologies by non-linear material based directional switch are considered as a possible long-term route in the evolution of current telecommunication network and ultra high-speed signal processing[10-17].

The all optical implementation of various subtraction schemes[5] have been studied by taking the complement of the subtrahend and adding it to the minuend. The schemes for optical implementation of subtractors using Semiconductor Optical Amplifier (SOA)[6] and electro-opticmodulators (EOM)[11] have been attempted. The paper presents a scheme for the implementation of all optical Parallel Subtractor (PS)[10] by Half Subtractor (HS) and Full Subtractor (FS)[15] with logic circuits in direct manner $[8,10]$, as done with paper and pencil, by nonlinear optical material based switching mechanism, in such a way that will obey the truth table[8,10,15]. By this method, each subtrahend bit of the number is subtracted from its corresponding significant minuend bit to get DIFFERENCE bit including the case of BORROW bit, as in conventional electronics. Our proposed circuits are very much reliable because they follow the basic principle of subtraction operation. An ALU of dream goal, an optical computer, can be implemented including this scheme.

\section{All-optical Switching Behaviour of Nonlinear Material and its Use as All-optical Gates}

The phenomenon photorefractivity[13-17] of some nonlinear optical material is used in nonlinear all-optical inten- 
sity switching mechanism. The photorefractive effect, where the refractive index changes induced by a light field when the crystal is subjected to intense laser radiation, defocusing and scattering of the light, is observed, as a result of an inhomogeneous change in the refractive index. It is also found that these changes still prevail even after the light is switched off, but it could be erased by strong, uniform illumination [14].The refractive index of some nonlinear materials (NLM) such as carbon disulfide, pure silica, potassium dihydrophosphate $(\mathrm{KDP}),\left(\mathrm{KH}_{2} \mathrm{PO}_{4}\right)$ crystal etc. varies linearly with the intensity of the light incident on it. The refractive index (n) of such isotropic dielectric non-crystalline media can be put into an equation as in (1).

$$
\mathrm{n}=\mathrm{n}_{0}+\mathrm{n}_{1} \mathrm{I}
$$

Here $\mathrm{n}_{0}$ is the linear term, $\mathrm{n}_{1}$ is the nonlinear correction term and $\mathrm{I}$ is the intensity of the incident light beam on the material.

We can implement the switching mechanism with such nonlinear material by taking an interface between two media of which one is a linear material (LM), whose refractive index $\mathrm{n}_{0}$ is independent of the intensity of light and the other is aforesaid NLM. A laser beam, highly intense polarized light, preferably pulse laser of intensity $\mathrm{I}_{1}$, is allowed to fall on the interface from linear to nonlinear part in a particular direction XO (incidence angle $\theta_{1}$ ) as depicted in Figure 1. The refracted beam from the NLM follows the path OZ. But when another higher intense laser beam of intensity $I_{2}\left(I_{2}>\right.$ $\mathrm{I}_{1}$ ) is made to incident along $\mathrm{XO}$, after refraction from the NLM the light passes through OY direction (angle of refraction $\theta_{2}$ ). The deviation of refractive angle for different incident light intensity $I_{1}$ and $I_{2}$ is $<Z O Y=\Delta \theta_{2}$. Thus the combination of LM and NLM may act nicely as a directional all-optical switch. This is the unit block of our proposed subtraction circuit.

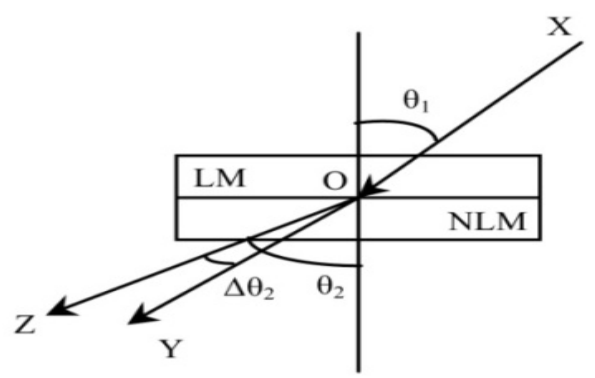

Figure 1. Intensity switching of optical nonlinear material

In the expression of refractive index in (1), $\mathrm{n}_{0}$ is linear term and $n_{1}$ is the nonlinear correction term. For carbon disulfide $\left(\mathrm{CS}_{2}\right)[2,3,12] \mathrm{n}_{0}=1.63, \mathrm{n}_{1}=514 \times 10^{-20} \mathrm{~m}^{2} / \mathrm{W}$. and for fused silicon dioxide[2,3,12] $\left(\mathrm{SiO}_{2}\right) \mathrm{n}_{0}=1.458, \mathrm{n}_{1}=$
$2.7 \times 10^{-20} \mathrm{~m}^{2} / \mathrm{W}$. If we use $\mathrm{CS}_{2}$ and $\mathrm{SiO}_{2}$ as nonlinear materials and the pulse laser of intensity $\mathrm{I}=2 \times 10^{18} \mathrm{~W} / \mathrm{m}^{2}$ as a source, we can estimate the deviations of light in two cases as given in Table 1.

The logic gates[2-3,5,15-17] are implemented in optics using NLM by taking the presence of light signal as 1 and the absence of it as 0 . The implementation of such logic gates can be done by using some femtosecond laser pulses and 1-mm-thick potassium dihydrophosphate crystal at the pick intensity of $0.6 \mathrm{TW} / \mathrm{cm}^{2}$ and duration of $60 \mathrm{fs}[3-4,15-17]$

\subsection{All Optical NOT Gate}

To implement an all optical NOT gate using non-linear material a constant intensity pulse laser source (CILS) is used as shown in Figure 2. It is also called probe beam. Here $\mathrm{P}_{1}$ is taken as input beam. A detector is placed at $\mathrm{P}_{2}$ will detect the output beam after refraction. If $\mathrm{P}_{1}$ is absent, the light will follow a path $\mathrm{OP}_{2}$ and will be detected by the detector due to presence of CILS. But if $\mathrm{P}_{1}$ is present, after refraction, the light will follow a path other than $\mathrm{OP}_{2}$, may be $\mathrm{OP}_{3}$, and the detector will not detect any light signal. Thus the system acts as optical NOT gate.

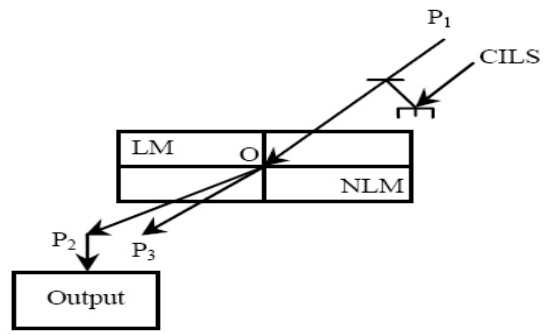

Figure 2. All-optical NOT gate

\subsection{All-optical Ex-OR Gate}

The two inputs all-optical XOR gate using NLM is shown in Figure 3. Here V1 and V2 are two input channels. A detector is placed at V3 gives the output. When only one input channel carries light signal, the light beam after refraction will detect by the detector at V3, unless not.

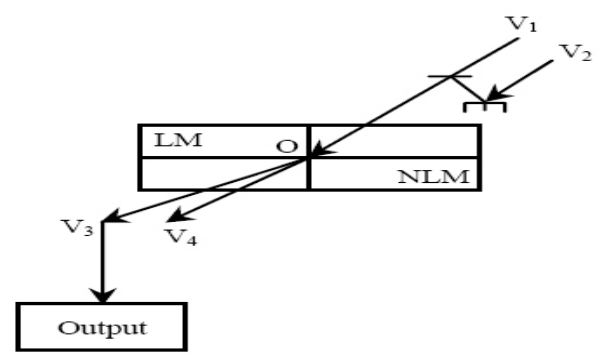

Figure 3. All-optical EX-OR gate

Table 1. Estimation of the Deviation of Pulsed Laser Light when Passing Through Carbon Disulfide $\left(\mathrm{CS}_{2}\right)$ and Silicon Dioxide $\left(\mathrm{SiO}_{2}\right)$

\begin{tabular}{cccccc}
\hline \hline Material & Angle of Incidence $\left(\theta_{1}\right)$ & Incident light intensity & $\mathrm{n}\left(=\mathrm{n}_{0}+\mathrm{n}_{1} \mathrm{I}\right)$ & Angle of refraction $\left(\theta_{2}\right)$ & Deviation $\left(\Delta \theta_{2}=\theta_{2}^{\prime}-\theta^{\prime \prime}{ }_{2}\right)$ \\
\hline \multirow{2}{*}{ carbon disulfide $\left(\mathrm{CS}_{2}\right)$} & $45 \mathrm{deg}$ & $\mathrm{I}=2 \times 10^{18} \mathrm{~W} / \mathrm{m}^{2}$ & 11.91 & $3.404 \mathrm{deg}=\theta_{2}^{\prime}$ & $1.578 \mathrm{deg}$ \\
& $45 \mathrm{deg}$ & $2 \mathrm{I}$ & 22.19 & $1.827 \mathrm{deg}=\theta^{\prime \prime}{ }_{2}$ & \multirow{2}{*}{$1.041 \mathrm{deg}$} \\
\hline \multirow{2}{*}{ Silicon dioxide $\left(\mathrm{SiO}_{2}\right)$} & $45 \mathrm{deg}$ & $\mathrm{I}=2 \times 10^{18} \mathrm{~W} / \mathrm{m}^{2}$ & 1.512 & $27.883 \mathrm{deg}=\theta_{2}^{\prime}$ & $27.842 \mathrm{deg}=\theta_{2}^{\prime \prime}$ \\
& $45 \mathrm{deg}$ & $2 \mathrm{I}$ & 1.566 & 276 \\
\hline \hline
\end{tabular}




\subsection{All Optical AND Gate}

The two inputs all-optical AND gate using NLM is shown in Figure 4. Here $R_{1}$ and $R_{2}$ are two input channels. A detector is placed at $R_{4}$ gives the output. Now when both the channels carry light signal, the light beam after refraction one can get light at the detector (situated at $\mathrm{R}_{4}$ ), unless not.

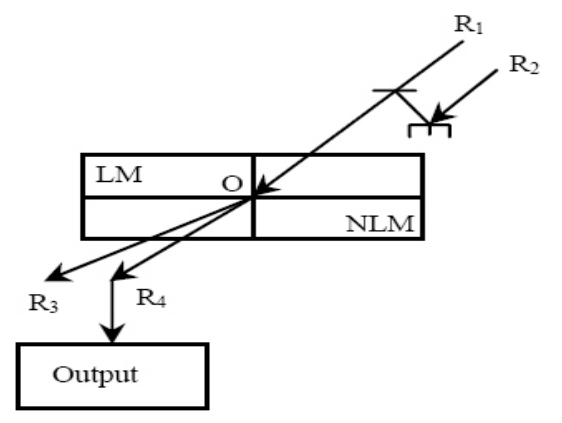

Figure 4. All-optical two-input AND gate

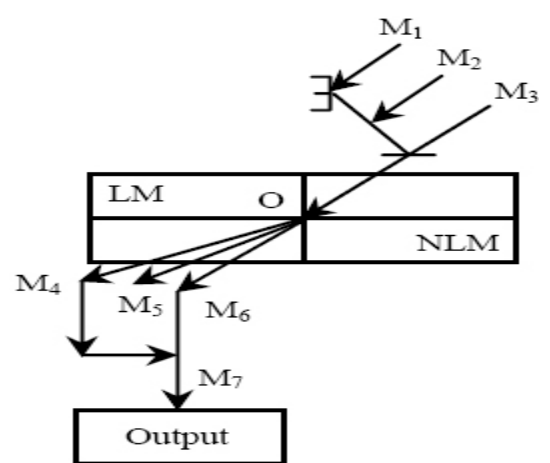

Figure 5. All optical three-input one-or-all circuit

\subsection{All Optical Three-input One-or-all Circuit}

The all-optical three-input one-or-all circuit using NLM is shown in Figure 5. Here $\mathrm{M}_{1}, \mathrm{M}_{2}$ and $\mathrm{M}_{3}$ are three input channels. A detector is placed at $\mathrm{M}_{7}$ gives the output. Now when any one of the input channels carries light signal, the light beam after refraction will detected by the detector at $\mathrm{M}_{7}$ and there will be light at $\mathrm{M}_{7}$ if all the inputs have light also The output remains dark for all other combinations of input states.

\subsection{All Optical Three-input Two-or-all Circuit}

The all-optical three-input two-or-all circuit using NLM is described in Figure 6. Here $\mathrm{N}_{1}, \mathrm{~N}_{2}$ and $\mathrm{N}_{3}$ are three input channels. A detector is placed at $\mathrm{N}_{7}$ gives the output. Now when any two or all of the input channels carry light signal, the light beam after refraction will detected by the detector at $\mathrm{M}_{7}$, unless not.

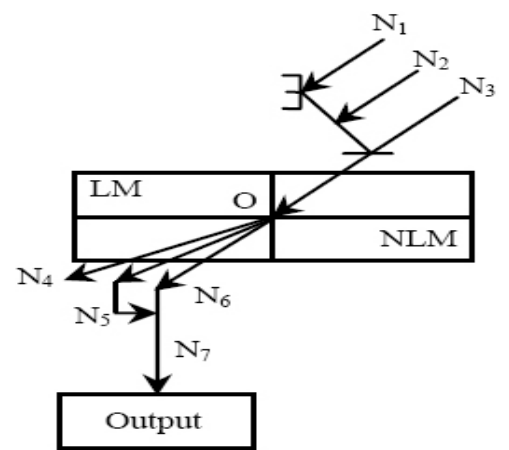

Figure 6. All optical three-input two-or-all circuit

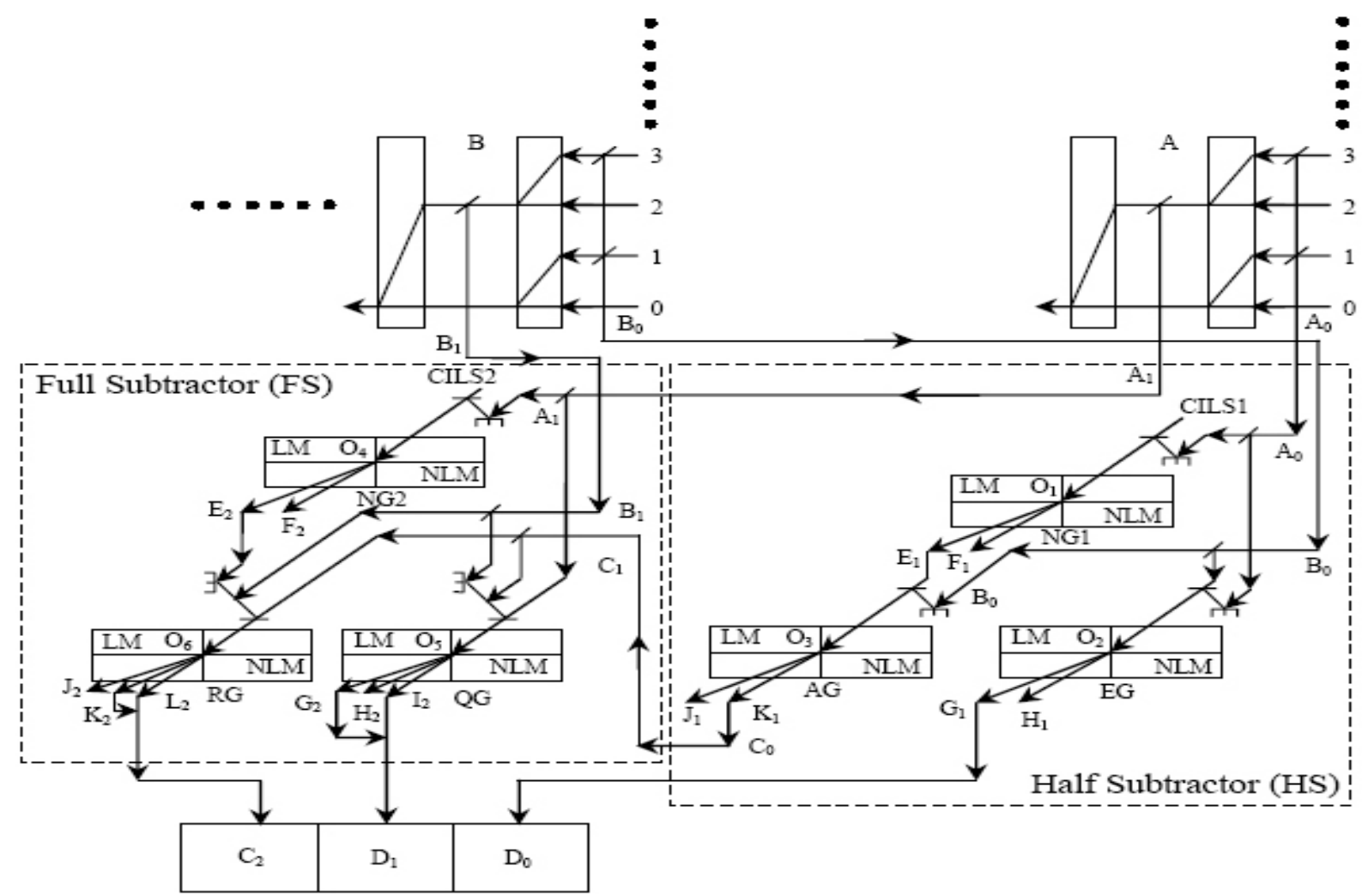

Figure 7. All-optical parallel subtraction schem using NLM as switch 


\section{All-Optical Parallel Subtractor}

We design an all-optical binary subtraction scheme capable of subtraction between two multibit data in parallel. The system is shown in the Figure 7 . Tree architectures[1,5,9] A and $B$ are used to convert a decimal value into its respective binary value, $A_{1} A_{0}$ and $B_{1} B_{0}$ respectively. Here $B_{1} B_{0}$ is to be subtracted from $A_{1} A_{0}$. One 1-bit full subtractor[15] and one 1-bit half subtractor[15] system are combined to obtain a general all optical parallel subtraction. For half subtractor system[15] $A_{0}$ and $B_{0}$ are the two inputs. The outputs are taken from $\mathrm{D}_{0}$ (DIFFERENCE) and $\mathrm{C}_{0}$ (BORROW). The all optical half subtractor is constructed by three all optical logic gates. NG1, EG and AG are the optical NOT gate, two-input EX-OR gate and two-input AND gate respectively. The truth table of an all-optical half subtractor is given in Table $2[8,10,15]$. For full subtractor[15], the inputs are $A_{1}, B_{1}$ and $\mathrm{C}_{1}\left(=\mathrm{C}_{0}\right.$ BORROW from previous half subtractor). The outputs are from $\mathrm{D}_{1}$ (DIFFERENCE) and $\mathrm{C}_{2}$ (BORROW). The all optical full subtractor is implemented with an all optical NOT gate (NG2), a three-input one_or_all circuit (QG) and a three-input two_or_all circuit (RG). The truth table of an all-optical full subtractor is set in Table 3 $[8,10,15]$. The final output result is $C_{2} D_{1} D_{0}$. This is the binary output of $\left(A_{1} A_{0}-B_{1} B_{0}\right)$. Let us consider an example for subtracting 3 from 2 . We take $A=2$ and $B=3$. The binary equivalent of $A=2$ is $A_{1} A_{0}=10$ and the binary equivalent of $\mathrm{B}=3$ is $\mathrm{B}_{1} \mathrm{~B}_{0}=11$.

Table 2. Truth Table of All-optical Half Subtractor

\begin{tabular}{cccc}
\hline \multicolumn{2}{c}{ Inputs } & \multicolumn{2}{c}{ Outputs } \\
\hline $\mathrm{A}_{0}$ & $\mathrm{~B}_{0}$ & $\mathrm{C}_{0}$ & $\mathrm{D}_{0}$ \\
\hline 0 & 0 & 0 & 0 \\
0 & 1 & 1 & 1 \\
1 & 0 & 0 & 1 \\
1 & 1 & 0 & 0 \\
\hline \hline
\end{tabular}

For half subtractor, the inputs are $A_{0}=0$ and $B_{0}=1$. The outputs will be DIFFERENCE $=1$ and $\mathrm{BORROW}=1$, which means $D_{0}=C_{0}=1$. Now the inputs of the full subtractor are $\mathrm{A}_{1}=1, \mathrm{~B}_{1}=1$ and $\mathrm{C}_{1}=1$ (from half subtractor BORROW). The output will be DIFFERENCE $=1$ and $\mathrm{BORROW}=1$ which means $\mathrm{D}_{1}=1$ and $\mathrm{C}_{2}=1$.

The final result will be $\mathrm{C}_{2} \mathrm{D}_{1} \mathrm{D}_{0}=111$ which comes from the subtraction of 11(3) from 10(2) when a 1 has been borrowed from the next stage. And then $10-11$ becomes $110(6)$ $-11(3)$ which gives $D_{1} D_{0}=11(3)$. The $C_{2}=1$ represents that 1 has to be deducted from next bit of the minuend.

For parallel subtraction of two different strings of multibit (more than 2 bits) binary number we need more expanded trees for decimal number to equivalent binary number conversion. And then, we also require additional full subtractors. The dots in the Figure 7 are representing such elevations.

All-optical parallel subtractor can subtract decimal or binary optical numbers in parallel. In our scheme we use all optical intensity switchs in designing the all optical parallel subtractor. In our design the light beam which is fed back is coming from the output of $\mathrm{O}_{1}$ and $\mathrm{O}_{4}$ switches. Again the concept used here to design these all optical switches $\left(\mathrm{O}_{1}\right.$ and $\mathrm{O}_{4}$ ) has an advantage. Whenever the outputs of these all optical switches $\left(\mathrm{O}_{1}\right.$ and $\left.\mathrm{O}_{4}\right)$ are assumed to be at ' 1 ' state, the source of that ' 1 ' state are the constant intensity pulse laser sources (CILS) used as probe beams. So in each feedback arrangement described in our scheme similar intense light beam is fed back. In this way the reduction of intensity by using beam splitter will not affect the non-linear response of the device. The light sources are so chosen that each input beam intensity is in the range of intensity which is detected as ' 1 ' by the detector after refraction.

Table 3. Truth Table of All_optical Full Subtractor

\begin{tabular}{ccccc}
\hline \hline & Inputs & \multicolumn{3}{c}{ Outputs } \\
\hline $\mathrm{A}_{\mathrm{n}}$ & $\mathrm{B}_{\mathrm{n}}$ & $\mathrm{C}_{\mathrm{n}-1}$ & $\mathrm{C}_{\mathrm{n}}$ & $\mathrm{D}_{\mathrm{n}}$ \\
\hline 0 & 0 & 0 & 0 & 0 \\
0 & 0 & 1 & 1 & 1 \\
0 & 1 & 0 & 1 & 1 \\
0 & 1 & 1 & 1 & 0 \\
1 & 0 & 0 & 0 & 1 \\
1 & 0 & 1 & 0 & 0 \\
1 & 1 & 0 & 0 & 0 \\
1 & 1 & 1 & 1 & 1 \\
\hline \hline
\end{tabular}

\section{Conclusions}

Our proposed circuits are very much reliable because they follow the basic principle of subtraction operation. The light signals that are severally used, bended and the feedback light signals from the outputs are made by mirrors and beam splitters to make the circuits simple. As the circuit is purely all-optical in nature and very simple, one can obtain the speed of operation far more than $\mathrm{THz}$ limit[2,3,12-24]. This subtraction scheme should be the first step on our dream way to all-optical Arithmetic Unit. The entire scheme should perform properly using suitable nonlinear material[2,3, 12-17,24]. Essentially inputs should be chosen properly for proper function of the system.

\section{ACKNOWLEDGEMENTS}

The authors want to acknowledge Professor Sourangshu Mukhopadhyay of the University of Burdwan for his valuable suggestions.

\section{REFERENCES}

[1] K. R. Choudhury and S. Mukhopadhyay, "A new method of binary addition scheme with massive use of non-linear material based system," Chinese Optics Letters, vol. 1, no. 4, pp. 132, 2003

[2] S. Dhar and S. Sahu, "All-optical implementation of S-R, clocked S-R and D flip-flops using nonlinear material," Opt. Eng. 47(6), 065401-1-6, 2008

[3] S. Sahu and S. Dhar, "Implementation of clocked J-K, T and 
J-K Master Slave flip-flops with nonlinear material in All-optical Domain,” Opt. Eng. 48(7), 075401-1-7, 2009

[4] S. Mironov. V. Lozhkarev, V. Ginzburg and E. Khazanov, "High-efficiency second-hermonic generation of superintense ultrashort laser pulses," Applied Optics. 48, 2051-2057, 2009

[5] K. R. Choudhury and S. Mukhopadhyay, "Binary optical arithmetic operation scheme with tree architecture by proper accommodation of optical nonlinear materials," Optical Engineering, vol. 43, no. 1, pp. 132, 2004

[6] Li Pei-Li, Huang De-Xiu, Zhang Xin-Liang and Wang Yang, "Single-SOA-Based Ultrahigh-Speed All-Optical Half Subtracter with PolSK Modulated Signals," Chinese Physics Letters, vol. 25, no. 5, pp. 1705, 2008

[7] S. K. Garai and S. Mukhopadhyay, "All-optical frequency encoded binary half subtractor using periodically poled lithium niobate waveguide and reflecting semiconductor optical amplifier," Optics and Photonics Letters, vol. 3, no. 1, pp. 15,1010

[8] M. Morris Mano, Digital Logic and Computer Design (Prentice-Hall of India Private Limited, New Delhi, 2000)

[9] S. Mukhopadhyay, "An optical conversion system: from binary to decimal and decimal to binary," Optics Communications, vol. 76, pp. 309, 1990

[10] R. P. Jain, Modern Digital Electronics, $3^{\text {rd }}$ ed., Tata MaGraw-Hill India, New Delhi. India: 2007

[11] A. Sinha, H. Bhowmik, P. Kuila, and S. Mukhopadhyay, "A new method of controlling the power of a gaussion optical pulse through electro-optic modulator and non-linear wave guide for generation of soliton," Optical Engineering, vol. 44, no. 6 , pp. 065003,2005

[12] D Samanta, S. Mukhopadhyay, "A method of maintaining the intensity level of a polarization encoded light signal," Journal of Physical Science. 11, 87-91, 2007

[13] S. D. Smith, I. Janossy, H. A. Mackenzi, J.G. H. Mathew, J. J. E. Reid, M. R. Taghizadeh, F. A. P. Tooley and A. C. Walker, "Nonlinear optical circuit elements, logic gates for optical computers: the first digital optical circuits," Optical Engi- neering, vol. 24, no. 4, pp. 569-573, 1985

[14] D. Arivouli, "Fundamentals of optical nonlinear materials," Pramana. 57(5-6) 871-883, 2001

[15] S. Sahu, R. R. Pal and S. Dhar, "A Novel Method of Implementing Nonlinear Material Based All-Optical Binary Half Subtractor and Full Subtractor System," J. of Electron Devices. 10, 493-498, 2011

[16] S. Sahu, R. R. Pal and S. Dhar, "TeraHertz All-Optical Binary Register using D flip-flop with Non-linear Material: A Proposal" J. of Electron Devices. 11, 588-595, 2011

[17] S. Sahu, R. R. Pal and S. Dhar, "All-Optical Binary Counter by using T flip-flop: An Implementation" International Journal of Engineering, Science and Technology. 3(10), 7799-7807, 2011

[18] A. Yang, Z. Liu, B. Wang, Y. Zhang and S. Liu, "Optical stream-cipher-like system for image encryption based on Michelson interferometer," Opt. Exp. 19(3), 2634-2642, 2011

[19] E. Lazzeri, G. Berrettini, G. Meloni, A. Bogoni and L. Poti, "All-Optical-Bits Shift Register Exploiting a Ring Buffer Based on Semiconductor Optical Amplifier," Phot. Tech. Lett. IEEE. 23(1), 45-47, 2011

[20] G. Berrettini, L. Potì and A. Bogoni, "Optical Dynamic RAM for All-Optical Digital Processing," Photonics Technology Letters, IEEE. 23(11), 685-687, 2011

[21] N. Pahari and S. Mukhopadhyay, "An all optical R-S flip-flop by optical non-linear material," J. of Opts. 34 (3), 108114, 2005

[22] A. K. Datta and S. Munshi, "Optical implementation of flip-flops using single-LCD panel," Opt. \& Laser Tech. 39(9), 2321-2329, 2007

[23] T. Chattopadhyay, "All-optical clocked delay flip-flop using a single terahertz optical asymmetric demultiplexer-based switch: a theoretical study," Appl. Opt. 49, 5226-5235, 2010

[24] M. Choi, S. H. Lee, Y. Kim, S. B. Kang, J. Shin, M. H. Kwak, K.Y. Kang, Y. H. Lee, N. Park and B. Min, "A terahertz metamaterial with unnaturally high refractive index," Nature. $470,369-373,2011$ 